\title{
SETS WITH FIXED POINT PROPERTY FOR ISOMETRIC MAPPINGS
}

\author{
ANTHONY TO-MING LAU ${ }^{1}$
}

\begin{abstract}
A subset $K$ of a Banach space $E$ is said to have the fixed point property for isometric mappings (f. p. p.) if there exists $z$ in $K$ such that $U(z)=z$ for each isometric mapping $U$ from $K$ onto $K$. We prove that any bounded closed subsets of $E$ with uniform relative normal structure have the f. p. p. We also prove that if $E$ is either $\mathscr{B}(H)$ (bounded operators on a Hilbert space $H$ ) or $l_{\infty}(X)$ (bounded functions on $X$ ), then $E$ is finite dimensional if and only if each weak*-compact convex subset of $E$ has the f. p. p. This is also equivalent to the convex set of (normal) states on $E$ having the f. p. p.
\end{abstract}

1. Introduction. A mapping $U: K \rightarrow E$ of a set $K$ in a Banach space $E$ into $E$ is called isometric if $\|U x-U y\|=\|x-y\|$ for all $x, y \in K$. We say that a subset $K$ of $E$ has the fixed point property for isometric mappings, or simply f. p. p., if there exists $z$ in $K$ such that $U(z)=z$ for any isometric mapping $U$ of $K$ onto itself. A well-known result of Brodskii and Milman [1] asserts that if $K$ is a weakly compact convex subset of $E$ and $K$ has normal structure, then $K$ has the f. p. p. In particular, any compact convex subset of $E$ has the f. p. p. (see [2, Lemma 1]).

We consider in this paper bounded closed subsets of a Banach space $E$ with the f. p. p. We prove, among other things, that any bounded closed subsets of $E$ with uniform relative normal structure have the f. p. p. We also show that if $E$ is either the space of bounded complex valued functions on a nonempty set $X$ with the supremum norm (denoted by $l_{\infty}(X)$ ), or the algebra of bounded operators on a Hilbert space $H$ (denoted by $\mathscr{B}(H)$ ), then $E$ is finite dimensional if and only if the set of states (or normal states) on $E$ has the f. p. p. This is also equivalent to each weak*-compact convex space of $E$ has the f. p. p. Our proofs depend on several well-known properties of discrete amenable groups.

2. Preliminaries and some notations. If $E$ is the Banach space $l_{\infty}(X)$ or $\mathscr{B}(H)$ as defined in $\S 1$, then $E$ has a unique predual $E_{*}$ (which is $l_{1}(X)$ in case $E=l_{\infty}(X)$, see [11] for details). A linear functional $\phi$ on $E$ is called a state if $\phi$ is positive and $\|\phi\|=1 ; \phi$ is normal if $\phi \in E_{*}$.

If $G$ is a group, then $G$ is amenable if there exists a state $\phi$ on $l_{\infty}(G)$ such that $\phi\left(l_{a} f\right)=\phi(f)$ for each $a \in G$, where $l_{a} f(X)=f(a x), x \in G$. As is well known, any group containing the free group on two generators is not amenable (see [8, p. 236]).

Received by the editors July 9, 1979.

AMS (MOS) subject classifications (1970). Primary 47H10; Secondary 43 A07.

Key words and phrases. Uniform relative normal structure, isometric mappings, fixed point property, invariant means on groups, operators on Hilbert space.

${ }^{1}$ This research is supported by NRC-A-7679. 
If $E$ is a Banach space, then the closed ball centre at $x \in E$ and radius $r>0$ will be denoted by $B[x, r]$. We list below some subsets of $E$ that are known to have the fixed point property:

(1) Any compact convex sets in $E$.

(2) Any closed ball $B=B\left[x_{0}, r\right]$. In this case $x_{0}$ is a common fixed point for any isometric mapping $U$ from $B$ onto $B$. Indeed, if $U\left(x_{0}\right)=x, x \neq x_{0}$, define $y=x_{0}$ $-t\left(x_{0}-x\right)$, where $t=r /\left\|x_{0}-x\right\|$, then $y \in B$ and $\|x-y\|=\left\|x-x_{0}\right\|+r>$ $r$. However, if $w \in B$ is such that $U(w)=y$, then $\|x-y\|=\left\|U\left(x_{0}\right)-U(w)\right\|=$ $\left\|x_{0}-w\right\|<r$, which is impossible.

(3) Any weakly compact convex set $K$ in $E$ if $E$ is strictly convex. In this case, any isometric mapping from $K$ onto $K$ is necessarily affine [3, Proposition 2]. Apply now the Ryll-Nardzewski fixed point theorem [7, p. 98].

It is not known whether there exist a weakly compact convex subset $K$ of a Banach space and a fixed point free isometry of $K$ onto $K$ (see [4] for a discussion of this open problem).

3. The main results. A closed subset $K$ of a Banach space $E$ is said to have uniform relative normal structure if there exists $0<c<1$ such that for any nonvoid bounded closed subset $M$ in $K$, there exist $z_{M}$ in $K$ such that

(i) $\left\|x-z_{M}\right\|<c \delta(M)$ for each $x \in M$,

(ii) if $y \in K$ such that $\|x-y\|<c \delta(M)$ for all $x \in M$, then

$$
\left\|z_{M}-y\right\|<c \delta(M)
$$

(Here $\delta(M)$ denotes the diameter of $M$.) The notion of uniform relative normal structure was introduced recently by $P$. M. Soardi [11]. He proved, among other things, that if $K$ is a nonempty weak*-closed set with uniform relative normal structure, and $T: K \rightarrow K$ is nonexpansive and leaves invariant a weak*-compact subset $M$ in $K$, then $K$ contains a fixed point for $T$. Examples of sets with uniform relative normal structure include any closed balls in $L^{\infty}(X, \delta, \mu)$ of a $\sigma$-finite measure space $(X, \delta, \mu)$ (see $[11$, p. 28]).

THEOREM 1. Let $E$ be a Banach space and let $K$ be a bounded closed nonempty subset of $E$ with uniform relative normal structure, then $K$ has the f.p.p.

Proof. Let $\mathscr{U}$ denote the group of isometric mappings from $K$ onto $K$ and let $0<c<1$ be a real number satisfying conditions (i) and (ii) for uniform relative normality of $K$. Put $A_{0}=K$, and let $r=\delta(K)$. If $r=0$, the conclusion is trivial. Otherwise define

$$
A_{1}=\left\{k \in A_{0} ; A_{0} \subseteq B[k, c r]\right\}
$$

Then $A_{1}$ is a closed nonempty subset of $K$ since $A_{1}$ is the intersection of $A_{0}$ with closed balls and $z_{A_{0}} \in A_{1}$ by (i). Also $U\left(A_{1}\right)=A_{1}$ for each $U \in \mathcal{Q}$. Indeed, if $h \in A_{1}$ and $x \in A_{0}$, let $y \in A_{0}$ such that $U(y)=x$. Then

$$
\|U(h)-x\|=\|U(h)-U(y)\|=\|h-y\|<c r .
$$


Hence $U\left(H_{0}\right) \subseteq H_{0}$. Equality follows by replacing $U$ with $U^{-1}$. Moreover $\delta\left(A_{1}\right)<$ cr. Hence the set

$$
H_{1}=\left\{k \in K ; A_{1} \subseteq B\left[k, c^{2} r\right]\right\}
$$

is again closed, nonempty and $U\left(H_{1}\right)=H_{1}$ for each $U \in \mathcal{Q}$. Define

$$
A_{2}=\left\{h \in H_{1} ; H_{1} \subseteq B\left[h, c^{2} r\right]\right\}
$$

Then $A_{2}$ is closed, nonempty (since $z_{A_{1}} \in A_{2}$ by condition (ii)) $\delta\left(A_{2}\right)<c^{2} r$ and $U\left(A_{2}\right)=A_{2}$ for each $U \in \mathcal{Q}$. Repeating this process, we have defined a sequence of nonempty closed sets $\left\{A_{n}\right\}_{n=1}^{\infty}$ in $K$ with the following properties:

(i) $U\left(A_{n}\right)=A_{n}$ for each $U \in \mathcal{U}$,

(ii) $\delta\left(A_{n}\right)<c^{n} r$,

(iii) $\|x-y\|<c^{n} r$ if $x \in A_{n-1}$ and $y \in A_{n}$.

For each $n=1,2, \ldots$, pick $z_{n} \in A_{n}$. Then, as readily checked, $\left\{z_{n}\right\}$ is Cauchy, and $U(z)=z$ for each $U \in \mathcal{Q}$ if $z$ is the limit point of $\left\{z_{n}\right\}$ in $K$.

THEOREM 2. Let $\boldsymbol{H}$ be a Hilbert space. Then the following are equivalent:

(a) $H$ is finite dimensional.

(b) The set of states on $\mathscr{B}(H)$ has the f.p.p.

(c) The set of normal states on $\mathscr{B}(H)$ has the f.p.p.

(d) Each weak*-compact convex subset of $\mathscr{B}(H)$ has the f.p.p.

Proof. If $H$ is finite dimensional, then each of the sets in (b), (c) and (d) is compact and convex, and hence has the f. p. p.

Let $\mathcal{Q}(H)$ denote the group of unitary elements in $\mathscr{B}(H)$. For each $u \in \mathcal{U}(H)$, define a weak*-weak ${ }^{*}$ continuous isometric linear map from $\mathscr{B}(H)$ onto $\mathscr{B}(H)$ by $\tau_{u}(x)=u^{*} x u, x \in \mathscr{B}(H)$. If $H$ is infinite dimensional, write $H=l_{2}(G)$ where $G$ is a free group, and $|G|=$ the cardinality of a complete orthonormal basis for $H$. For each $g \in G$, the operator $l_{g}: l_{2}(G) \rightarrow l_{2}(G)$ defined by $l_{g} h(t)=h(g t), t \in G$, is in थ(H).

Note that each of the sets in (b) and (c) is invariant under each $\tau_{u}^{*}, u \in \mathcal{U}(H)$. Hence if (b) or (c) holds, then there exists a state $\phi$ on $\mathscr{B}(H)$ such that $\tau_{u}^{*} \phi=\phi$ for each $u \in \mathcal{Q}(H)$. For each $f \in l_{\infty}(G)$, define $x_{f}: l_{2}(G) \rightarrow l_{2}(G)$ by $x_{f}(h)=f \cdot h$ (pointwise multiplication), $h \in l_{2}(G)$. Then $\left\|x_{f}\right\|<\|f\|$. Define $m \in l_{\infty}(G)^{*}$ by

$$
m(f)=\phi\left(x_{f}\right), \quad f \in l_{\infty}(G) .
$$

Then, as readily checked, $m$ is a state on $l_{\infty}(G)$. Also, if $g \in G, f \in l_{\infty}(G)$ then $l_{g} x_{f} l_{g^{-1}}=x_{k}$ where $k=l_{g} f$. Hence

$$
m(f)=\phi\left(x_{f}\right)=\phi\left(l_{g} x_{f} l_{g-1}\right)=m\left(l_{g} f\right),
$$

i.e. $G$ is amenable, which is impossible. Hence $H$ is finite dimensional.

Finally if (d) holds, and $H$ is infinite dimensional, let $V N(G)$ denote the weak*-closure of the linear span of $\left\{l_{g} ; g \in G\right\}$ in $\mathscr{B}(H)$. For each $x \in \mathscr{B}(H)$, let $K(x)$ be the weak*-closure of the convex hull of $\left\{l_{g-1} x l_{g}, g \in G\right\}$. Then $K(x)$ is weak*-compact and invariant under each $\tau_{u}$, when $u=l_{g}, g \in G$. Hence there exists $z \in K(x)$ such that $l_{g^{-1}} z l_{g}=z$ for each $g \in G$. In particular, $z$ is in the 
commutant of $V N(G)$. By [10, Proposition 4.4.21], $G$ must be amenable, which is impossible.

THEOREM 3. Let $X$ be a nonempty set. Then the following are equivalent:

(a) $X$ is finite.

(b) The set of states on $l_{\infty}(X)$ has the $f . p . p$.

(c) The set of normal states on $l_{\infty}(X)$ has the $f . p . p$.

(d) Each weak*-compact convex subset of $l_{\infty}(X)$ has the $f . p . p$.

Proof. If $X$ is finite, then $l_{\infty}(X)$ and $l_{\infty}(X)^{*}$ are finite dimensional, and hence (b), (c) and (d) hold.

If $X$ is infinite, then we may regard $X$ as a free group $G$ on $|X|$-generators. Also, the set of states (or normal states) on $l_{\infty}(G)$ is invariant under each of the isometrics $l_{g}^{*}, g \in G$. Hence if (b) or (c) holds, then there exists a state $\phi$ on $l_{\infty}(G)$ such that $l_{g}^{*} \phi=\phi$ for all $g \in G$, which is impossible. Finally, if (d) holds, then for each $f \in l_{\infty}(G)$, define $\left(r_{g} f\right)(x)=f(x g)$ for each $x, a \in G$. Let $K(f)$ be the weak*-closed convex hull of $\left\{r_{g} f ; g \in G\right\}$. Then $K(f)$ is also weak*-compact. Hence by (d), there exists $h \in K(f)$, such that $r_{g} h=h$ for all $g \in G$. Necessarily $h$ is a constant. By a result of Mitchell [9, Corollary 2], $G$ is amenable.

Theorem 4. Let $X$ be a nonempty set. Then the following are equivalent:

(a) $X$ has one element.

(b) The set of nonzero multiplicative linear functionals on $l_{\infty}(X)$ has the $f . p \cdot p$.

(c) Each weak*-compact subset of $l_{\infty}(X)$ has the f.p.p.

Proof. That (a) implies (b) and (c) is trivial. To prove (b) $\Rightarrow$ (c) and (c) $\Rightarrow$ (a), regard $X$ as a group. Then the set of nonzero multiplicative linear functionals on $l_{\infty}(G)$ is invariant under each of the isometric mappings $l_{g}^{*}, g \in G$. Now if (b) holds, then there exists a nonzero multiplicative linear functional $\phi$ on $l_{\infty}(G)$ such that $l_{g}^{*} \phi=\phi$ for all $g \in G$, which implies $G$ is trivial by [5, Corollary 3]. If (c) holds, then an argument similar to that of Theorem 3 shows that for each $f$ in $l_{\infty}(G)$, the weak*-closure of $\left\{r_{g} f ; g \in G\right\}$ contains a constant, which again implies that $G$ is trivial by [6, Theorem 1] and [5, Corollary 3].

Remark. Let $E$ be a dual Banach space, then each closed ball in $E$ is weak*compact convex and has the f. p. p. Also, if $E$ is finite dimensional, then each weak*-compact convex subset of $E$ has the f. p. p. However, the converse is false unless $E=l_{\infty}(X)$, or $E=\mathscr{B}(H)$ by Theorems 2 and 3. (For example, take $E$ to be an infinite dimensional Hilbert space.)

\section{REFERENCES}

1. M. S. Brodskii and D. P. Milman, On the center of a conoex set, Dokl. Akad. Nauk SSSR 59 (1948), 838-840. (Russian)

2. R. DeMarr, Common fixed points for commuting contraction mappings, Pacific J. Math. 13 (1963), 1139-1141.

3. M. Edelstein, On non-expansive mappings of Banach spaces, Proc. Cambridge Philos. Soc. 60 (1964), 439-447.

4. _ Remarks and questions concerning non-expansive mappings, Fixed Point Theory and its Application, edited by S. Swaminathan, Academic Press, New York, 1976, pp. 63-67.

5. E. Granirer, Extremely amenable semigroups, Math. Scand. 17 (1965), 117-197. 
6. __ Extremely amenable semigroups. II, Math. Scand. 20 (1967), 93-113.

7. F. P. Greenleaf, Inoariant means on topological groups, Van Nostrand Math. Studies, no. 16, Van Nostrand Reinhold and Winston, Princeton, N. J., 1969.

8. E. Hewitt and K. Ross, Abstract harmonic analysis. I, Springer-Verlag, Berlin, 1963.

9. T. Mitchell, Constant functions and left-invariant means on semigroups, Trans. Amer. Math. Soc. 119 (1965), 244-261.

10. S. Sakai, $C^{*}$-algebras and $W^{*}$-algebras, Springer-Verlag, Berlin, 1971.

11. P. M. Soardi, Existence of fixed points of non-expansive mappings in certain Banach spaces, Proc. Amer. Math. Soc. 73 (1979), 25-29.

Departmient of Mathematics, University of Alberta, Edmonton, Alberta T6g 2G1, Canada 